Homology, Homotopy and Applications, vol.9(1), 2007, pp.257-274

\title{
COMPACTLY GENERATED HOMOTOPY CATEGORIES
}

\author{
HENRIK HOLM AND PETER JØRGENSEN
}

(communicated by Luchezar Avramov)

\begin{abstract}
Over an associative ring we consider a class $\mathbb{X}$ of left modules which is closed under set-indexed coproducts and direct summands. We investigate when the triangulated homotopy category $\mathrm{K}(\mathbb{X})$ is compactly generated, and give a number of examples.
\end{abstract}

\section{Introduction}

Let $\mathbb{X}$ be a class of left $R$-modules which is closed under set-indexed coproducts and direct summands. When the (triangulated) homotopy category $\mathrm{K}(\mathbb{X})$ associated to $\mathbb{X}$ is compactly generated, it can be a powerful tool. Let us mention two recent examples from the literature to illustrate this point.

In $[\mathbf{2 3}$, Thm. 2.4] it is proved that the homotopy category $\mathrm{K}(\operatorname{Proj} R)$ is compactly generated provided that $R$ is coherent from either side, and that every flat left $R$ module has finite projective dimension.

The result above is the cornerstone in proving that the class of Gorenstein projective modules is precovering (also called contravariantly finite) when $R$ is commutative and noetherian with a dualizing complex ([22, Cor. 2.13]). The question of whether the Gorenstein projective modules really do constitute a precovering class has been studied by many people; see, for example, $[\mathbf{5}, \mathbf{1 1}, \mathbf{1 3}, \mathbf{1 8}, \mathbf{3 2}]$.

In [25, Prop. 2.3] it is shown that $\mathrm{K}(\operatorname{Inj} R)$ is compactly generated when $R$ is left noetherian, and in [19, Cor. 5.5] this result is applied to give a new and interesting characterization of Gorenstein rings in terms of (totally) acyclic complexes of injective modules.

In this paper we study the general question: When is $\mathrm{K}(\mathbb{X})$ compactly generated? More precisely, we give a number of sufficient conditions on $R$ and $\mathbb{X}$ which ensure that $\mathrm{K}(\mathbb{X})$ is compactly generated, and our results generalize those of $[\mathbf{2 3 , 2 5}$. At this point it is worth mentioning that the innocent-looking $\mathrm{K}(\operatorname{Mod} \mathbb{Z})$ is known not to be compactly generated ([29, Lem. E.3.2]).

Our main result is Theorem 3.1, given in Section 3. Sections 4 and 5 develop the necessary machinery to provide us with examples where Theorem 3.1 can be applied. In the final Section 6 we use the previous results to list a number of concrete classes $\mathbb{X}$ for which $\mathrm{K}(\mathbb{X})$ is compactly generated.

Received September 5, 2006, revised November 16, 2006; published on February 12, 2007. 2000 Mathematics Subject Classification: 16D20, 16D40, 16D50, 16D90, 16 E05.

Key words and phrases: Compactly generated category, compact object, homotopy category, pure exact sequence, triangulated category.

Copyright (C) 2007, International Press. Permission to copy for private use granted. 
Homology, Homotopy and Applications, vol.9(1), 2007

\section{Preliminaries}

The assumptions, the notation, and the definitions from this section will be used throughout the paper.

Definition 2.1 (Setup). Throughout, $R$ is a ring, and all modules are left $R$-modules unless otherwise specified. We use $R^{\text {op }}$ to denote the opposite ring of $R$, and a left $R^{\mathrm{op}}$-module is naturally identified with a right $R$-module.

The symbol $\mathbb{X}$ always denotes a class of modules with Add $\mathbb{X}=\mathbb{X}$ (Definition 2.2 below). For an arbitrary class of modules we write $\mathbb{A}$.

Definition 2.2 (Notation). We shall frequently use the following categories:

- $\operatorname{Mod} R$ is the category of all $R$-modules, and $\bmod R$ is the category of all finitely presented $R$-modules.

- $\operatorname{Proj} R, \ln R$, and Flat $R$ are the categories of projective, injective, and flat $R$-modules respectively.

- PureProj $R$ and Purelnj $R$ are the categories of pure projective and pure injective $R$-modules respectively ([21, App. A $]$ ).

- For a class of modules $\mathbb{A}$, we write add $\mathbb{A}$ for the category of modules which are isomorphic to a direct summand of a module of the form $\coprod_{i \in I} A_{i}$, where $A_{i} \in \mathbb{A}$ and $I$ is a finite set. Allowing arbitrary index sets $I$, in this construction we get $\operatorname{Add} \mathbb{A}$.

The homotopy category. Let $\mathbb{A}$ be a class of modules with $\mathbb{A}=\operatorname{add} \mathbb{A}$. The objects of the homotopy category $\mathrm{K}(\mathbb{A})$ are chain complexes of modules from $\mathbb{A}$. Even though $\mathbb{A}$ is not abelian, the notion of complexes is still well-defined since the condition $\partial^{2}=0$ makes sense. The morphisms of $\mathrm{K}(\mathbb{A})$ are chain maps modulo homotopy equivalence. By, for example [33, Chap. 10], $\mathrm{K}(\mathbb{A})$ carries the structure of a triangulated category with finite coproducts. If $\mathbb{A}$ has arbitrary set-indexed coproducts, then so has $\mathrm{K}(\mathbb{A})$.

Definition 2.3. Let $T$ be a triangulated category $([\mathbf{2 9}])$, closed under set-indexed coproducts. An object $C \in \mathrm{T}$ is compact if the natural map

$$
\coprod_{i \in I} \operatorname{Hom}_{\mathrm{T}}\left(C, X_{i}\right) \longrightarrow \operatorname{Hom}_{\mathrm{T}}\left(C, \coprod_{i \in I} X_{i}\right)
$$

is an isomorphism for any family $\left\{X_{i}\right\}_{i \in I}$ of objects in $\mathrm{T}$. A set of objects $\mathcal{G} \subseteq \mathrm{T}$ is called a generating set if the implication

$$
\operatorname{Hom}_{\mathrm{T}}(G, X)=0 \text { for all } G \in \mathcal{G} \Longrightarrow X \cong 0
$$

holds for all $X \in \mathrm{T}$. If $\mathrm{T}$ has a generating set consisting of compact objects, then $\mathrm{T}$ is called compactly generated.

Example 2.4. The derived category $\mathrm{D}(\operatorname{Mod} R)$ of the abelian category $\operatorname{Mod} R$ is always compactly generated by the set

$$
\mathcal{G}=\left\{\Sigma^{n} R \mid n \in \mathbb{Z}\right\} .
$$

Here $R$ is considered as a complex concentrated in degree zero with zero differentials, and $\Sigma^{n}: \mathrm{D}(\operatorname{Mod} R) \longrightarrow \mathrm{D}(\operatorname{Mod} R)$ denotes the $n$-th shift "to the left", that is, for a 
complex

$$
X=\cdots \longrightarrow X_{s+1} \stackrel{\partial_{s+1}^{X}}{\longrightarrow} X_{s} \stackrel{\partial_{s}^{X}}{\longrightarrow} X_{s-1} \longrightarrow \cdots,
$$

the complex $\Sigma^{n} X$ has the module $X_{t-n}$ in degree $t$ and $(-1)^{n} \partial_{t-n}^{X}$ as its $t^{\prime}$ th differential.

Surprisingly, the corresponding homotopy category $\mathrm{K}(\operatorname{Mod} R)$ is not even compactly generated when $R=\mathbb{Z}$; see $[\mathbf{2 9}$, Lem. E.3.2]. The ring $\mathbb{Z}$ has pure global dimension 1 (Example 5.7(1)). It is a consequence of the theory developed in this paper (Section 6(3)) that $\mathrm{K}(\operatorname{Mod} R)$ is compactly generated when $R$ has pure global dimension 0 .

Remark 2.5. For the notion of compact, that is, $\aleph_{0}$-compact objects, the reader is referred to Neeman [29, Chap. 4.1 and 4.2]. The definition of a generating set is taken from [29, Def. 8.1.1]. Definition 2.3 only has interest for us in the case where $\mathrm{T}=\mathrm{K}(\mathbb{X})$ for some class of modules $\mathbb{X}$ (Definition 2.1).

\section{A condition for compact generatedness}

In this section we give conditions on the module category $\mathbb{X}$ (Definition 2.1) which ensure that the associated homotopy category $\mathrm{K}(\mathbb{X})$ is compactly generated (Definition 2.3). We begin by stating our main result, but we postpone the proof until the end of the section where the necessary preparations have been made.

Theorem 3.1. Let $\mathbb{X}=\operatorname{Add} \mathbb{X}$ be a class of $R$-modules, and assume that every finitely presented module $M$ has a right $\mathbb{X}$-resolution $X(M)$. Then

$$
\mathcal{G}_{\mathbb{X}}=\left\{\Sigma^{n} X(M) \mid M \in \bmod R, n \in \mathbb{Z}\right\}
$$

is a set of compact objects in $\mathrm{K}(\mathbb{X})$. Furthermore, $\mathcal{G}_{\mathbb{X}}$ generates $\mathrm{K}(\mathbb{X})$ if and only if every pure exact sequence in $\operatorname{Mod} R$, consisting of modules from $\mathbb{X}$, is split exact.

How to apply Theorem 3.1. In order to apply the theorem above we need examples of classes $\mathbb{X}=\operatorname{Add} \mathbb{X}$ satisfying:

(a) Every finitely presented module has a right $\mathbb{X}$-resolution.

(b) Every pure exact sequence in $\operatorname{Mod} R$, consisting of modules from $\mathbb{X}$, is split exact.

In Section 4 we give examples of classes satisfying condition (a), and in Section 5 we discuss how to check whether (b) holds. In the final Section 6 we use the results from the previous sections to list a number of concrete classes $\mathbb{X}$ for which $\mathrm{K}(\mathbb{X})$ is compactly generated.

Before proving Theorem 3.1 we need some preparation, in particular some remarks about right resolutions and pure exactness.

Right resolutions. Let $\mathbb{A}$ be a class of modules. An $\mathbb{A}$-preenvelope of a module $M$ 
is a homomorphism $\varphi: M \longrightarrow A$ with $A \in \mathbb{A}$, such that given any other homomorphism $\varphi^{\prime}: M \longrightarrow A^{\prime}$ with $A^{\prime} \in \mathbb{A}$ there is a (not necessarily unique) factorization,

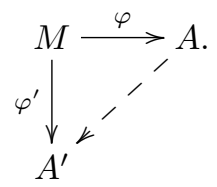

Let $M$ be a module which has an $\mathbb{A}$-preenvelope, $\varphi^{0}: M \longrightarrow A^{0}$. Suppose that the cokernel of this map, $\operatorname{Coker}\left(\varphi^{0}\right)$, also has an $\mathbb{A}$-preenvelope, say, $\varphi^{1}: \operatorname{Coker}\left(\varphi^{0}\right) \longrightarrow$ $A^{1}$. If also $\operatorname{Coker}\left(\varphi^{1}\right)$ has an $\mathbb{A}$-preenvelope etc., we may construct an augmented right $\mathbb{A}$-resolution of $M$,

$$
0 \longrightarrow M \stackrel{\varepsilon}{\longrightarrow} A^{0} \stackrel{\partial^{0}}{\longrightarrow} A^{1} \stackrel{\partial^{1}}{\longrightarrow} A^{2} \stackrel{\partial^{2}}{\longrightarrow} \cdots .
$$

Here $\varepsilon=\varphi^{0}$, and $\partial^{n}$ is the composition

$$
A^{n} \longrightarrow \operatorname{Coker}\left(\varphi^{n}\right) \stackrel{\varphi^{n+1}}{\longrightarrow} A^{n+1} .
$$

The complex $(*)$ is not necessarily exact (as $\mathbb{A}$-preenvelopes are not necessarily injective), however $\operatorname{Hom}_{R}\left((*), A^{\prime}\right)$ is exact for every $A^{\prime} \in \mathbb{A}$. There is a useful equivalent way of stating this property of the complex $(*)$, namely if we consider the chain map

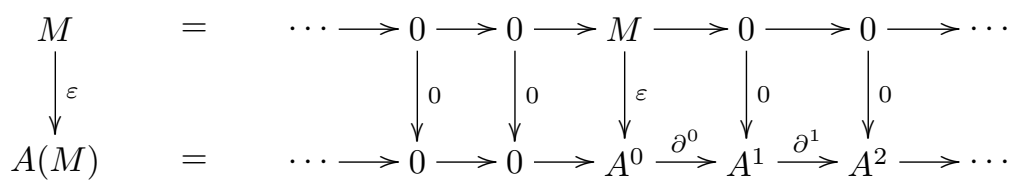

then $\operatorname{Hom}_{R}\left(\varepsilon, A^{\prime}\right)$ is a quasi-isomorphism for all $A^{\prime} \in \mathbb{A}$. In the given situation we refer to

$$
A(M)=0 \longrightarrow A^{0} \stackrel{\partial^{0}}{\longrightarrow} A^{1} \stackrel{\partial^{1}}{\longrightarrow} A^{2} \stackrel{\partial^{2}}{\longrightarrow} \cdots
$$

as a (non-augmented) right $\mathbb{A}$-resolution of $M$. Finally, the class $\mathbb{A}$ is called preenveloping (also known as covariantly finite) if every module has an $\mathbb{A}$-preenvelope, and thus a right $\mathbb{A}$-resolution.

If a module $M$ admits a right $\mathbb{A}$-resolution, it is in general not unique. However, by, for example [11, Ex. 8.1.3], all possible choices of right $\mathbb{A}$-resolutions $A(M)$ of $M$ are homotopy equivalent and thus isomorphic in $\mathrm{K}(\mathbb{A})$. Hence $A(M)$ is a well-defined object in the homotopy category of $\mathbb{A}$. As the chain map $\varepsilon: M \longrightarrow A(M)$ becomes a quasi-isomorphism whenever the functor $\operatorname{Hom}_{R}\left(-, A^{\prime}\right)$ is applied to it for $A^{\prime} \in \mathbb{A}$, and since both $M$ and $A(M)$ are left-bounded complexes, [8, Prop. (2.7)(b)] implies that

$$
\operatorname{Hom}_{R}\left(A(M), A^{\prime}\right) \underset{\operatorname{Hom}_{R}\left(\varepsilon, A^{\prime}\right)}{\simeq} \operatorname{Hom}_{R}\left(M, A^{\prime}\right)
$$

is a quasi-isomorphism for every complex $A^{\prime}$ consisting of modules from $\mathbb{A}$. In 
particular we have an equivalence of functors $\mathrm{K}(\mathbb{A}) \longrightarrow \operatorname{Mod} \mathbb{Z}$,

$$
\mathrm{H}_{0} \operatorname{Hom}_{R}(A(M),-) \simeq \mathrm{H}_{0} \operatorname{Hom}_{R}(M,-) \text {. }
$$

For the discussion above we do not assume that $\mathbb{A}$ has set-indexed coproducts, however since we are interested in compact objects in the homotopy category, we will focus on the case where $\mathbb{A}=\mathbb{X}$ (Definition 2.1).

Proposition 3.2. If a module $M$ admits a right $\mathbb{X}$-resolution $X(M)$, then there is an equivalence of functors $\mathrm{K}(\mathbb{X}) \longrightarrow \operatorname{Mod} \mathbb{Z}$,

$$
\operatorname{Hom}_{\mathrm{K}(\mathbb{X})}(X(M),-) \simeq \mathrm{H}_{0} \operatorname{Hom}_{R}(M,-) .
$$

In particular, if $M$ is finitely generated and admits a right $\mathbb{X}$-resolution, then $X(M)$ is a compact object in $\mathrm{K}(\mathbb{X})$.

Proof. We have natural equivalences of functors,

$$
\begin{aligned}
\operatorname{Hom}_{\mathrm{K}(\mathbb{X})}(X(M),-) & \simeq \mathrm{H}_{0} \operatorname{Hom}_{R}(X(M),-) \\
& \simeq \mathrm{H}_{0} \operatorname{Hom}_{R}(M,-),
\end{aligned}
$$

where the first isomorphism is standard, and the second is, by $(\dagger)$ above. For the last claim in the proposition we use that $\mathrm{H}_{0} \operatorname{Hom}_{R}(M,-)$ commutes with set-indexed coproducts if $M$ is finitely generated.

Definition 3.3 (Pure exact sequences). A (not necessarily short) sequence $Y$ in $\operatorname{Mod} R$ is pure exact if and only if $\operatorname{Hom}_{R}(M, Y)$ is exact for all finitely presented modules $M$ ([21, Thm. 6.4]).

Proof of Theorem 3.1. By Proposition 3.2, the set $\mathcal{G}_{\mathbb{X}}$ consists of compact objects. Strictly speaking, $\mathcal{G}_{\mathbb{X}}$ is not a set, as $\bmod R$ is not. However, we may of course restrict ourselves to just looking at isomorphism classes in $\bmod R$, and they do constitute a set.

Now let $Y$ be an arbitrary object in $\mathrm{K}(\mathbb{X})$, that is, a chain complex of modules from $\mathbb{X}$. We claim that the following conditions are equivalent:

(i) $\operatorname{Hom}_{\mathrm{K}(\mathbb{X})}\left(\Sigma^{n} X(M), Y\right)=0$ for all $M \in \bmod R$ and $n \in \mathbb{Z}$;

(ii) $Y$ is a pure exact sequence in $\operatorname{Mod} R$.

Having proved this, the last part of the theorem follows immediately, since an object $Y \in \mathrm{K}(\mathbb{X})$ is isomorphic to zero if and only if $Y$ splits $([33$, Ex. 1.4.3]). The proof of the equivalence of (i) and (ii) follows from Definition 3.3 compared with the following calculation:

$$
\begin{aligned}
\operatorname{Hom}_{\mathrm{K}(\mathbb{X})}\left(\Sigma^{n} X(M), Y\right) & \cong \operatorname{Hom}_{\mathrm{K}(\mathbb{X})}\left(X(M), \Sigma^{-n} Y\right) \\
& \cong \mathrm{H}_{0} \operatorname{Hom}_{R}\left(M, \Sigma^{-n} Y\right) \\
& \cong \mathrm{H}_{n} \operatorname{Hom}_{R}(M, Y),
\end{aligned}
$$

where the second isomorphism is by Proposition 3.2. 


\section{Existence of right resolutions}

In this section we study constructions and examples of module classes $\mathbb{X}$ (Definition 2.1) for which every finitely presented module has a right $\mathbb{X}$-resolution. This is of interest when we want to apply our main Theorem 3.1. For reasons which will become clear in Propositions 5.1 and 5.2, we will only focus on such classes which have the additional property that they are contained in either Purelnj $R$ or PureProj $R$ (Definition 2.2).

We begin by stating all our constructions and examples 4.1-4.7, but we postpone the arguments to the end of the section.

Example 4.1. The following two examples are classical:

(a) The class $\ln \mathrm{R}$ is preenveloping by [31, Thm. 3.13 and 3.26]. However, in order for $\operatorname{lnj} R$ to be closed under coproducts, $R$ must be left noetherian ([31, Thm. 4.27]).

(b) The class Purelnj $R$ is preenveloping by [21, Prop. 7.6]. However, in order for Purelnj $R$ to be closed under coproducts, $R$ must be pure-semisimple, and in this case we actually have Purelnj $R=\operatorname{Mod} R([21$, Thm. B.18]).

In fact, Example 4.1(a) admits the following generalization. In the result below, $\operatorname{lnj}\left(M_{1}, \ldots, M_{n}\right)$ is defined as in Definition 4.8 .

Proposition 4.2. Assume that $R$ is left noetherian and let $M_{1}, \ldots, M_{n}$ be $R$ bimodules such that each $M_{j}$ is finitely generated as a left $R$-module. Then every module has a right $\operatorname{Inj}\left(M_{1}, \ldots, M_{n}\right)$-resolution.

Next we present examples which are contained in PureProj $R$.

Example 4.3. The following conclusions hold:

(a) Every finitely presented module has a right PureProj $R$-resolution.

(b) If $R$ is right coherent, then every finitely presented module has a right resolution with respect to $\operatorname{Add}\left({ }_{R} R\right)=\operatorname{Proj} R$.

Actually, Example 4.3(b) admits a generalization:

Proposition 4.4. Assume that $R$ is right coherent, and let $M$ be an $R$-bimodule which is finitely presented from either side. Then every finitely presented module has a right $\operatorname{Add}\left({ }_{R} M\right)$-resolution.

In the next result, Gproj $R$ denotes the class of finitely generated Gorenstein projective modules $([\mathbf{2}, \mathbf{3}])$, and GFlat $R$ is the class of Gorenstein flat modules ([12]). Furthermore, $\lim G \operatorname{croj} R$ is the class of modules which can be written as a colimit in $\operatorname{Mod} R$ of some functor $I \longrightarrow$ Gproj $R$, where $I$ is a small filtering category (Definition 4.12).

Proposition 4.5. Assume that $R$ is commutative and noetherian with a dualizing complex. If $\lim$ Gproj $R=$ GFlat $R$ (this happens, for example, if $R$ also has finite injective dimension over itself), then every finitely presented module has a right $\operatorname{Add}(\operatorname{Gproj} R)$-resolution. 
In the next result, sub $\mathbb{A}$ is defined as in Lemma 4.14 .

Proposition 4.6. Let $\mathbb{A}$ be a class of modules, and assume that every finitely presented module $N$ has an $\mathbb{A}$-preenvelope $\varphi: N \longrightarrow A$ for which the image $\operatorname{Im}(\varphi)$ is finitely presented. Then every finitely presented module has a right $\operatorname{Add}(\operatorname{sub} \mathbb{A})$ resolution.

Example 4.7. To apply Proposition 4.6 above, we first need a class $\mathbb{A}$ such that every finitely presented module has an $\mathbb{A}$-preenvelope. For instance, $\mathbb{A}$ could be any of the following preenveloping classes:

(a) Purelnj $R \cap$ Flat $R$ if $R$ is right coherent ([11, Prop. 6.6.6]).

(b) Flat $R$ if $R$ is right coherent ([11, Prop. 6.5.1]).

(c) The class of $S$-torsion free modules, when $R$ is commutative and $S \subseteq R$ is a multiplicative subset (see below).

But other choices of $\mathbb{A}$ are also possible; for example from the proof of Proposition 4.4 it will follow that:

(d) If $R$ is right coherent, and $M$ is an $R$-bimodule which is finitely presented from either side, then every finitely presented module has an $\operatorname{add}\left({ }_{R} M\right)$-preenvelope (note add, not Add).

However, $\mathbb{A}$ must have the additional property that among all the preenvelopes of a given finitely presented module $N$, there should exist one with a finitely presented image. We note that

(1) If $R$ is left noetherian, then the image of every $\mathbb{A}$-preenvelope of $N$ is finitely presented.

(2) If $\mathbb{A} \subseteq \bmod R$ (this is the case in (d) above) and $R$ is left coherent, then the image of every preenvelope of $N$ is finitely presented by [11, Thm. 3.2.24].

Before proving 4.2-4.6 we will get 4.7 (c) out of the way:

Torsion-free modules. Let $R$ be commutative and let $S \subseteq R$ be a multiplicative subset. For any module $M$, its $S$-torsion submodule is defined as

$$
\Gamma_{S} M=\{x \in M \mid s x=0 \text { for some } s \in S\} .
$$

We say that $M$ is $S$-torsion-free if $\Gamma_{S} M=0$. It is easy to see that the class of $S$ torsion-free modules is preenveloping, as $M \longrightarrow M / \Gamma_{S} M$ is an $S$-torsion-free preenvelope of $M$.

In the rest of the section we prove Propositions 4.2-4.6. We begin with a definition.

Definition 4.8. Assume that $R$ is left noetherian and let $M_{1}, \ldots, M_{n}$ be $R$-bimodules such that each $M_{j}$ is finitely generated as a left $R$-module. A module $J$ belongs to $\operatorname{lnj}\left(M_{1}, \ldots, M_{n}\right)$ if and only if there exist injective modules $I_{1}, \ldots, I_{n}$ such that $J$ is a direct summand of

$$
\operatorname{Hom}_{R}\left(M_{1}, I_{1}\right) \oplus \cdots \oplus \operatorname{Hom}_{R}\left(M_{n}, I_{n}\right) .
$$


Proof of Proposition 4.2. As $R$ is left noetherian, there is, by $[\mathbf{1 1}$, Proof of Thm. 5.4.1], a set of injective modules $\mathbb{E}$ such that $\operatorname{Inj} R=\operatorname{Add} \mathbb{E}$. Using that $\operatorname{Hom}_{R}\left(M_{j},-\right)$ commutes with set-indexed coproducts, we see that $\operatorname{Inj}\left(M_{1}, \ldots, M_{n}\right)$ has the form Add $\mathbb{A}$, where

$$
\mathbb{A}=\left\{\operatorname{Hom}_{R}\left(M_{j}, E\right) \mid j \in\{1, \ldots, n\}, E \in \mathbb{E}\right\} .
$$

From the description in Definition 4.8 it is clear that $\operatorname{lnj}\left(M_{1}, \ldots, M_{n}\right)$ is closed under products, and hence it follows easily from $[\mathbf{1 1}, \operatorname{Prop} .6 .2 .1]$ that $\operatorname{Inj}\left(M_{1}, \ldots, M_{n}\right)$ is preenveloping.

Remark 4.9. Note that $\operatorname{Inj}\left(M_{1}, \ldots, M_{n}\right)$ is contained in Purelnj $R$.

Proof of Example 4.3. Part (a) is clear, as PureProj $R$ contains every finitely presented module. Part (b) follows from [10, Ex. 3.4].

Before we go on, we need a few facts about finitely presented modules.

Lemma 4.10. The following conclusions hold:

(a) If $M$ is a finitely presented module and $S \subseteq M$ a finitely generated submodule, then the quotient $M / S$ is finitely presented.

(b) If $M$ is finitely generated and $S \subseteq M$ is a submodule such that $M / S$ is finitely presented, then $S$ is finitely generated.

(c) Assume that $R$ is left coherent, $M$ is finitely generated, and $N$ is finitely presented. If $\varphi: M \longrightarrow N$ is a homomorphism, then $\operatorname{Ker}(\varphi)$ is finitely generated.

Proof. Part (a) is easy to prove, and part (b) can be found in, for example, [26, Prop. (4.26)(b)]. Using (b), we can easily prove (c) as follows.

As $M$ is finitely generated, then so is $\operatorname{Im}(\varphi)$. As $R$ is coherent and $\operatorname{Im}(\varphi)$ is a finitely generated submodule of the finitely presented module $N$, it follows by [26, Def. (4.51) and Cor. (4.52)] that $\operatorname{Im}(\varphi)$ is even finitely presented. Applying (b) to the inclusion $\operatorname{Ker}(\varphi) \subseteq M$, which has $M / \operatorname{Ker}(\varphi) \cong \operatorname{Im}(\varphi)$, we get that $\operatorname{Ker}(\varphi)$ is finitely generated.

Lemma 4.11. Assume that $\mathbb{A} \subseteq \bmod R$, and that every finitely presented module has an $\mathbb{A}$-preenvelope. Then every finitely presented module has a right Add $\mathbb{A}$-resolution.

Proof. Let $M$ be a finitely presented module, and let $\varphi^{0}: M \longrightarrow A^{0}$ be an $\mathbb{A}$-preenvelope. Since $\operatorname{Im}\left(\varphi^{0}\right)$ is finitely generated and $A^{0}$ is finitely presented, Lemma 4.10(a) implies that Coker $\left(\varphi^{0}\right)$ is finitely presented, so it has an $\mathbb{A}$-preenvelope, $\varphi^{1}: \operatorname{Coker}\left(\varphi^{0}\right) \longrightarrow A^{1}$. Continuing in this manner, we build an augmented right $\mathbb{A}$-resolution of $M$,

$$
A^{+}(M)=0 \longrightarrow M \longrightarrow A^{0} \longrightarrow A^{1} \longrightarrow \cdots \text {. }
$$

To finish the proof, it suffices to see that $\operatorname{Hom}_{R}\left(A^{+}(M), A^{\prime}\right)$ is exact for every $A^{\prime} \in \operatorname{Add} \mathbb{A}$. We may assume that $A^{\prime}$ has the form $\coprod_{i \in I} A_{i}$ where $A_{i} \in \mathbb{A}$. Finally, we simply have to use that

$$
\operatorname{Hom}_{R}\left(A^{+}(M), \coprod_{i \in I} A_{i}\right) \cong \coprod_{i \in I} \operatorname{Hom}_{R}\left(A^{+}(M), A_{i}\right)
$$

as every module in $A^{+}(M)$ is, in particular, finitely generated. 
Definition 4.12 (Modules with support in a category). For a class $\mathbb{A}=\operatorname{add} \mathbb{A}$ of finitely presented modules, Lenzing $[\mathbf{2 7}]$ introduces the class $\lim _{\mathbb{A}}$ of all colimits in $\operatorname{Mod} R$ of functors $I \longrightarrow \mathbb{A}$, where $I$ is a small filtering category $([\mathbf{2 8}$, Chap. IX]). A module in $\lim _{\longrightarrow} \mathbb{A}$ is said to have support in $\mathbb{A}$.

In [27, Prop. 2.1] two alternative characterizations of the modules with support in $\mathbb{A}$ are given. The following result can be found in, for example, [1, Thm. 3.2] or $[\mathbf{9}$, Sec. (4.2)].

Proposition 4.13. If $\mathbb{A} \subseteq \bmod R$ with $\operatorname{add} \mathbb{A}=\mathbb{A}$, then the following two conditions are equivalent:

(i) Every finitely presented module has an $\mathbb{A}$-preenvelope.

(ii) $\lim _{\longrightarrow} \mathbb{A}$ is closed under products.

The following proof is a consequence of the above proposition:

Proof of Proposition 4.5. Since $R$ is commutative and noetherian with a dualizing complex, [8, Thm. 5.7] gives that GFlat $R$ is closed under products. The assumption $\underline{\lim }(\operatorname{Gproj} R)=$ GFlat $R$, Proposition 4.13 and Lemma 4.11 applied to $\mathbb{A}=$ Gproj $R$

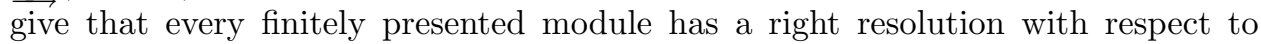
$\operatorname{Add}(\operatorname{Gproj} R)$.

It remains to prove the claim in parentheses, namely that the equality

$$
\underset{\lim }{\longrightarrow}(\operatorname{Groj} R)=\text { GFlat } R
$$

holds when $R$ is commutative and noetherian with finite injective dimension over itself. The inclusion " $\supseteq$ " follows from [11, Thm. 10.3.8]. The opposite inclusion " $\subseteq$ " follows from combining $[\mathbf{8}$, Thm. (3.5)] and [33, Cor. 2.6.17] with the fact that every module has finite Gorenstein flat dimension. For the latter claim, see for example $[8,(1.3)$ and Thm. (4.1)] or [7, Thm. (5.2.10)] in the local case.

Our next goal is to provide the proof of Proposition 4.4:

Proof of Proposition 4.4. As ${ }_{R} M$ is finitely presented, it suffices by Lemma 4.11 to show that every finitely presented module $N$ has an $\operatorname{add}\left({ }_{R} M\right)$-preenvelope. We start by proving that the $R^{\mathrm{op}}$-module $\operatorname{Hom}_{R}(N, M)$ is finitely generated: Since $N$ is finitely presented, there is an exact sequence,

$$
F_{1} \longrightarrow F_{0} \longrightarrow N \longrightarrow 0
$$

where $F_{i} \cong\left({ }_{R} R\right)^{b_{i}}$ is finitely generated and free. Applying the left exact functor $\operatorname{Hom}_{R}(-, M)$ to this sequence, we get

$$
0 \longrightarrow \operatorname{Hom}_{R}(N, M) \longrightarrow \operatorname{Hom}_{R}\left(F_{0}, M\right) \longrightarrow \operatorname{Hom}_{R}\left(F_{1}, M\right) .
$$

Since $\operatorname{Hom}_{R}\left(F_{i}, M\right) \cong\left(M_{R}\right)^{b_{i}}$, and since $M_{R}$ is finitely presented, we see that $\operatorname{Hom}_{R}\left(F_{i}, M\right)$ is finitely presented. Applying Lemma 4.10(c) to $(*)$ we get that 
Homology, Homotopy and Applications, vol. 9(1), 2007

$\operatorname{Hom}_{R}(N, M)$ is finitely generated, and we write

$$
\operatorname{Hom}_{R}(N, M)=h_{1} R+\cdots+h_{t} R .
$$

We claim that the map $\varphi: N \longrightarrow M^{t}$ defined by

$$
z \longmapsto\left(h_{1}(z), \ldots, h_{t}(z)\right)
$$

is an $\operatorname{add}\left({ }_{R} M\right)$-preenvelope of $N$. To see this, it suffices to prove that any homomorphism $\psi: N \longrightarrow M^{k}$ from $N$ to a finite power of ${ }_{R} M$ lifts to $M^{t}$,

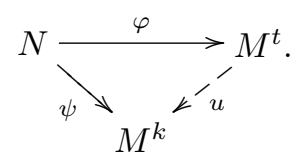

Furthermore, without loss of generality we may assume that $k=1$. To define $u$, use (**) to write $\psi \in \operatorname{Hom}_{R}(N, M)$ as

$$
\psi=h_{1} r_{1}+\cdots+h_{t} r_{t}
$$

for suitable $r_{1}, \ldots, r_{t} \in R$. We can then define $u: M^{t} \longrightarrow M$ by

$$
\left(x_{1}, \ldots, x_{t}\right) \longmapsto x_{1} r_{1}+\cdots+x_{t} r_{t} .
$$

Now $u \varphi=\psi$ because for $z \in N$ we have:

$$
\begin{aligned}
u \varphi(z) & =u\left(h_{1}(z), \ldots, h_{t}(z)\right) \\
& =h_{1}(z) r_{1}+\cdots+h_{t}(z) r_{t} \\
& =\psi(z)
\end{aligned}
$$

Finally we need to show Proposition 4.6, but first a little preparation:

Lemma 4.14. Let $\mathbb{A}$ be any class of modules and define

$$
\operatorname{sub} \mathbb{A}=\{S \in \bmod R \mid S \subseteq A \text { for some } A \in \mathbb{A}\} .
$$

Assume that $M$ is a finitely presented module, and that $M$ admits an $\mathbb{A}$-preenvelope $\varphi: M \longrightarrow A$ such that $\operatorname{Im}(\varphi)$ is finitely presented. Then $M$ has a right sub $\mathbb{A}$-preenvelope.

Proof. By assumption, $M$ has an $\mathbb{A}$-preenvelope $\varphi: M \longrightarrow A$ such that $\operatorname{Im}(\varphi)$ is finitely presented. Consider the obvious factorization

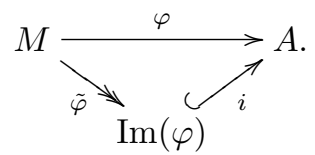

By definition, the module $\operatorname{Im}(\varphi)$ belongs to sub $\mathbb{A}$, and it is easy to verify that $\tilde{\varphi}: M \longrightarrow \operatorname{Im}(\varphi)$ is indeed a sub $\mathbb{A}$-preenvelope of $M$.

Proof of Proposition 4.6. The assumptions on $\mathbb{A}$ and Lemma 4.14 ensure that every finitely presented module has a sub $\mathbb{A}$-preenvelope. Since sub $\mathbb{A}$ is contained in $\bmod R$ by definition, Lemma 4.11 finishes the proof. 
Remark 4.15. The class $\operatorname{Add}(\operatorname{sub} \mathbb{A})$ is contained in PureProj $R$, since sub $\mathbb{A}$ is contained in $\bmod R$ by definition.

\section{When does a pure exact sequence split?}

Given a class of modules $\mathbb{A}$, we discuss in this section how to see whether every pure exact sequence in $\operatorname{Mod} R$, consisting of modules from $\mathbb{A}$, is split exact. This question is of interest when we wish to apply our main theorem, Theorem 3.1. We begin by outlining the idea of this section, but we postpone the arguments until later.

For any class of modules, we consider two conditions (PP) and (PI); see Definition 5.4. These conditions can be checked, and are indeed fulfilled in many cases as Proposition 5.2 below shows. The conditions (PP) and (PI) are the key ingredients in the following proposition, which is the main result of this section:

Proposition 5.1. Let $\mathbb{A}$ be a class of modules satisfying at least one of the two conditions (PP) or (PI). Then every pure exact sequence in $\operatorname{Mod} R$, consisting of modules from $\mathbb{A}$, is split exact.

Proposition 5.2. The conclusions below hold:

(a) If $R$ has finite left pure global dimension (Example 5.7), then every subclass of PureProj $R$ satisfies (PP), and every subclass of Purelnj $R$ satisfies (PI).

(b) If every flat $R$-module has finite projective dimension, then every subclass of Proj $R$ satisfies (PP).

(c) If $R$ is left noetherian, then every subclass of $\operatorname{Inj} R$ satisfies (PI).

The rest of the section is devoted to proving Propositions 5.1 and 5.2. We begin with the following:

Definition 5.3. For a class of modules $\mathbb{A}$ we define $F(\mathbb{A})$ to be the class of modules which are isomorphic to some kernel (equivalently, some image, or some cokernel) in a pure exact sequence,

$$
\cdots \longrightarrow A_{n+1} \longrightarrow A_{n} \longrightarrow A_{n-1} \longrightarrow \cdots
$$

where every $A_{n}$ belongs to Add $\mathbb{A}$.

The properties (PP) and (PI) for a class $\mathbb{A}$, which occur in this section's main result 5.1, are defined in terms of $F(\mathbb{A})$ from Definition 5.3:

Definition 5.4. For a class of modules $\mathbb{A}$ we consider the properties:

(PP) There exists a $d \geqslant 0$ such that for every $M \in \mathrm{F}(\mathbb{A})$ and every pure exact sequence $\quad 0 \rightarrow K_{d} \rightarrow A_{d-1} \rightarrow \cdots \rightarrow A_{0} \rightarrow M \rightarrow 0$ with $A_{0}, \ldots, A_{d-1} \in \mathbb{A}$, the module $K_{d}$ must be pure projective.

(PI) There exists a $d \geqslant 0$ such that for every $M \in \mathrm{F}(\mathbb{A})$ and every pure exact sequence $0 \rightarrow M \rightarrow A^{0} \rightarrow \cdots \rightarrow A^{d-1} \rightarrow C^{d} \rightarrow 0$ with $A^{0}, \ldots, A^{d-1} \in \mathbb{A}$, the module $C^{d}$ must be pure injective. 
The purpose of the following Remark 5.5 and the subsequent Lemma 5.6 is to get a better feeling for the construction $\mathrm{F}(-)$ from Definition 5.3.

Remark 5.5. Clearly, Add $\mathbb{A} \subseteq \mathrm{F}(\mathbb{A})$. Furthermore, if $\mathbb{A}=\operatorname{add} \mathbb{A}$ consists of finitely presented modules, then $\mathrm{F}(\mathbb{A}) \subseteq \lim _{\mathbb{A}}$ (Definition 4.12), since:

If $M \in \mathrm{F}(\mathbb{A})$, then in particular there exists a module $A \in \operatorname{Add} \mathbb{A}$ and a pure monomorphism $0 \rightarrow M \rightarrow A$. Since $A \in \lim \mathbb{A}$, and since $\lim \mathbb{A}$ is closed under pure submodules by [27, Prop. 2.2], it follows that $M$ belongs to $\stackrel{\lim }{\longrightarrow}$.

Lemma 5.6. The following conclusions hold:

(a) $\mathrm{F}(\operatorname{Proj} R) \subseteq$ Flat $R$. If $R$ is a commutative integral domain which is not a field, then the inclusion is strict.

(b) If $R$ is left noetherian, then $\mathrm{F}(\operatorname{Inj} R)=\operatorname{Inj} R$.

Proof. "(a)": It follows immediately from Definition 5.3 of $\mathrm{F}(-)$ that $\mathrm{F}($ Proj $R)=$ $\mathrm{F}(\operatorname{add} R)$, and the latter is contained in $\lim (\operatorname{add} R)=$ Flat $R$ by $\operatorname{Remark} 5.5$. If $R$ is a commutative integral domain with quotient field $Q \neq R$, then $Q$ belongs to Flat $R$, but $Q \notin \mathrm{F}(\operatorname{Proj} R)$, since $Q$ cannot even be embedded into a free module.

"(b)": Only the inclusion $\mathrm{F}(\operatorname{Inj} R) \subseteq \operatorname{lnj} R$ is non-trivial; thus we let $M \in \mathrm{F}(\operatorname{Inj} R)$ and use Baer's criterion to show that $M$ is injective. The assumption on $M$ implies, in particular, the existence of a pure epimorphism $f: I \longrightarrow M$, where $I$ is injective. Let $\mathfrak{a} \subseteq R$ be an ideal, and let $i: \mathfrak{a} \longrightarrow R$ be the inclusion. Given a homomorphism $u: \mathfrak{a} \longrightarrow M$, we must find $v: R \longrightarrow M$ with $v i=u$. Since $R$ is left noetherian, the ideal $\mathfrak{a}$ is finitely presented, so by assumption on $f$ we get $g: \mathfrak{a} \longrightarrow I$ with $f g=u$,

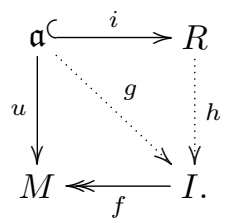

Injectivity of $I$ then gives $h: R \longrightarrow I$ with $h i=g$. Consequently, the homomorphism $v=f h: R \longrightarrow M$ is the desired one.

Krull dimension of categories. Geigle [15, Def. 2.1] has introduced a Krull dimension for a small additive category $\mathcal{C}$. By definition, the Krull-Geigle dimension of $\mathcal{C}$ coincides with the Krull-Gabriel dimension (introduced in [14] using filtrations of localizing subcategories) of

$$
\text { f.p.funct }\left(\mathcal{C}^{\text {op }}, A b\right) \text {. }
$$

The latter is the category of all covariant, additive, and finitely presented functors $\mathcal{C}^{\text {op }} \longrightarrow \mathrm{Ab}$, where $\mathrm{Ab}=\operatorname{Mod} \mathbb{Z}$.

For a ring $R$, Jensen-Lenzing [21, pp. 197-199] consider a Krull dimension for $\bmod \left(R^{\mathrm{op}}\right)$; by definition it is the Krull-Gabriel dimension of the category

$$
\text { f.p.funct }\left(\bmod \left(R^{\mathrm{op}}\right), \mathrm{Ab}\right) \text {. }
$$

For an Artin algebra $\Lambda$, there is by [4, Thm. 3.3] a duality, in other words a 
"contravariant equivalence",

$$
D: \bmod \Lambda \longrightarrow \bmod \left(\Lambda^{\mathrm{op}}\right) .
$$

Consequently, there is also an equivalence of categories,

$$
\begin{gathered}
\text { f.p.funct }\left(\bmod \left(\Lambda^{\mathrm{op}}\right), \mathrm{Ab}\right) \stackrel{\sim}{\longrightarrow} \text { f.p.funct }\left((\bmod \Lambda)^{\mathrm{op}}, \mathrm{Ab}\right) \\
F \quad \longmapsto \quad F \circ D .
\end{gathered}
$$

In particular, the Krull-Jensen-Lenzing dimension of $\bmod \left(\Lambda^{\mathrm{op}}\right)$ agrees with the Krull-Geigle dimension of $\bmod \Lambda$. By $[\mathbf{1 5}$, Thm. 4.3], the latter is finite when $\Lambda$ is a tame hereditary Artin algebra.

Example 5.7 (Pure global dimension). The definition of the left pure global dimension for a ring $R$, denoted l.p.gl.dim $R$, may be found in, for example [21, Def. A.14]. Below we give examples of classes of rings with finite left pure global dimension.

(1) If $\max \left\{\aleph_{0},|R|\right\}=\aleph_{t}$, then l.p.gl.dim $R \leqslant t+1$ by [17, Sec.2].

(2) If $I \subseteq R$ is a two-sided ideal, then l.p.gl.dim $R / I \leqslant 1$.p.gl.dim $R$. If $R$ is commutative and $S \subseteq R$ is a multiplicative subset, then l.p.gl.dim $S^{-1} R \leqslant$ l.p.gl.dim $R$; see [24, Prop. 1.1].

(3) If $(R, \mathfrak{m}, k)$ is a commutative local noetherian domain of Krull dimension 1, and $k$ is at most countable, then l.p.gl.dim $R=1$ by [20, Prop. 4.7].

(4) If $R$ is a finite dimensional $k$-algebra ( $k$ any field) of tame representation type, which is either hereditary or a radical-squared zero algebra, then l.p.gl.dim $R \leqslant$ 2 by [6, Prop. 3.3].

(5) Some specific examples of four-dimensional $k$-algebras (which are neither hereditary nor radical-squared zero) with finite pure global dimension may be found in [6, Prop. 5.1]. The reader might also want to consult [21, Cor. 11.33 and 11.34].

(6) If the category $\bmod \left(R^{\mathrm{op}}\right)$ has finite Krull dimension $d$ according to JensenLenzing [21, pp. 197-199] (see remarks above), then l.p.gl.dim $R \leqslant d$ by [21, Thm. 11.31]. This applies to the rings $R$, for example, where:

- $R$ is a Dedekind domain; [21, Thm. 8.55, Cor. 11.32].

- $R$ is a tame hereditary Artin algebra (see remarks above).

(7) If $R$ is von Neumann regular, then every exact sequence is pure exact, and therefore l.p.gl.dim $R$ equals the (ordinary) left global dimension of $R$. This applies to the rings $R$, for example:

- If $R$ has left global dimension zero, that is, $R$ is left semi-simple, then l.p.gl.dim $R=0$.

- The ring $R=\left\{\left(x_{n}\right)_{n \in \mathbb{N}} \in k^{\mathbb{N}} \mid x_{n}\right.$ constant for $\left.n \gg 0\right\}$ ( $k$ any field), is von Neumann regular with unit. Also, $R$ has global dimension 1: As $R$ is not noetherian, its global dimension is $>0$. Since $R$ is von Neumann regular, every ideal is generated by idempotents ${ }^{1}$. Clearly, $R$ has only $\aleph_{0}$ many idempotents, so [30, Cor. 2.47] implies the claim.

\footnotetext{
${ }^{1}$ This follows easily from the fact $[\mathbf{1 6}$, Thm. p. 10] that every principal ideal is generated by an idempotent.
} 
For later use, we note that a von Neumann regular ring is automatically coherent from either side.

Proof of Proposition 5.2. "(a)": In Definition 5.4 we may take $d$ to be the left pure global dimension $d$ of $R$.

"(b)": If every flat module has finite projective dimension, then there in fact exists $d$ such that $\operatorname{pd}_{R} F \leqslant d$ for all $F \in$ Flat $R$. If $\mathbb{A} \subseteq \operatorname{Proj} R$, we get $\mathrm{F}(\mathbb{A}) \subseteq$ Flat $R$ by Lemma 5.6(a), and it follows immediately that the number $d$ implements the (PP) property for $\mathbb{A}$.

"(c)": If $\mathbb{A} \subseteq \operatorname{Inj} R$, we get $\mathrm{F}(\mathbb{A}) \subseteq \operatorname{Inj} R$ by Lemma 5.6(b), and it follows that the number $d=0$ implements the (PI) property for $\mathbb{A}$.

Proof of Proposition 5.1. Let $A=\cdots \rightarrow A_{n+1} \rightarrow A_{n} \rightarrow A_{n-1} \rightarrow \cdots$ be a pure exact sequence with $A_{i} \in \mathbb{A}$, and decompose $A$ into short exact sequences

$$
S_{n}=0 \longrightarrow \Omega_{n} \longrightarrow A_{n} \longrightarrow \Omega_{n-1} \longrightarrow 0 .
$$

It follows that every $S_{n}$ is pure exact. We want to prove that $S_{n}$ is split exact, so it suffices to show that $\Omega_{n-1}$ is pure projective, or that $\Omega_{n}$ is pure injective. We will actually prove the following:

(a) If $\mathbb{A}$ has property (PP), then every $\Omega_{n}$ is pure projective.

(b) If $\mathbb{A}$ has property (PI), then every $\Omega_{n}$ is pure injective.

We will only prove (a), as the proof of (b) is similar. By Definition 5.3, every $\Omega_{m}$ belongs to $F(\mathbb{A})$. To see that $\Omega_{n}$ is pure projective, we consider the pure exact sequence

$$
0 \longrightarrow \Omega_{n} \longrightarrow A_{n} \longrightarrow \cdots \longrightarrow A_{n-d+1} \longrightarrow \Omega_{n-d} \longrightarrow 0,
$$

where $d$ is a number which implements the property (PP) for $\mathbb{A}$. Since $\Omega_{n-d}$ belongs to $\mathrm{F}(\mathbb{A})$, and $A_{n-d+1}, \ldots, A_{n}$ belong to $\mathbb{A}$, the property (PP) guarantees that $\Omega_{n}$ is pure projective.

\section{Summary}

Using the results from the previous sections we are now able to give a list of examples of concrete module classes $\mathbb{X}=\operatorname{Add} \mathbb{X}$ (Definition 2.1) such that the triangulated homotopy category $\mathrm{K}(\mathbb{X})$ is compactly generated. In most of our examples, rings with finite pure global dimension play an important role (Example 5.7).

(1) Assume that $R$ is right coherent with finite left pure global dimension, and that $M$ is an $R$-bimodule which is finitely presented from either side. Then we may take

$$
\mathbb{X}=\operatorname{Add}\left({ }_{R} M\right) .
$$

If ${ }_{R} M$ is projective, then $\operatorname{Add}\left({ }_{R} M\right) \subseteq \operatorname{Proj} R$, and the condition "finite left pure global dimension" may be replaced by "every flat module has finite projective dimension". Thus under this assumption, the special case $M=R$ recovers 
Homology, Homotopy and Applications, vol.9(1), 2007

[23, Thm. 2.4], namely we may take

$$
\mathbb{X}=\operatorname{Proj} R \text {. }
$$

References: 3.1, 4.4, 5.1, and 5.2(a)(b).

(2) Assume that $R$ is left noetherian with finite left pure global dimension, and that $M_{1}, \ldots, M_{n}$ are $R$-bimodules such that each $M_{j}$ is finitely generated as a left $R$-module. Then we may take

$$
\mathbb{X}=\operatorname{Inj}\left(M_{1}, \ldots, M_{n}\right) .
$$

If every $\left(M_{j}\right)_{R}$ is flat, then $\operatorname{Inj}\left(M_{1}, \ldots, M_{n}\right) \subseteq \operatorname{Inj} R$, and the condition "finite left pure global dimension" is superfluous. In particular, the special case $n=1$ and $M_{1}=R$ recovers [25, Prop. 2.3], namely we may take

$$
\mathbb{X}=\operatorname{lnj} R \text {. }
$$

References: $3.1,4.2,4.8,5.1$, and 5.2(a)(c).

(3) If $R$ has finite left pure global dimension then we may take

$$
\mathbb{X}=\text { PureProj } R \text {. }
$$

In particular, if $R$ is left pure-semisimple then we can use

$$
\mathbb{X}=\operatorname{Mod} R \text {. }
$$

References: 3.1, 4.3(a), 4.1(b), 5.1, and 5.2(a).

(4) Assume that $R$ is commutative and noetherian with a dualizing complex, and that $R$ has finite pure global dimension. If $\lim$ Groj $R=$ GFlat $R$ (this happens for example if $R$, in addition, has finite injective dimension over itself) then we may take

$$
\mathbb{X}=\operatorname{Add}(\operatorname{Gproj} R) .
$$

References: $3.1,4.5,5.1$, and 5.2(a).

(5) If $R$ is left noetherian and right coherent with finite left pure global dimension then we may take, for example,

$$
\mathbb{X}=\operatorname{Add}(\operatorname{sub}(\text { Flat } R)) \text {. }
$$

References: 3.1, 4.6, 4.7(b), 4.7(1), 5.1, and 5.2(a).

(6) If $R$ is commutative and noetherian with finite pure global dimension, and $S \subseteq R$ is a multiplicative subset then we may take

$$
\begin{aligned}
\mathbb{X} & =\operatorname{Add}(\operatorname{sub}\{S \text {-torsion free modules }\}) \\
& =\operatorname{Add}\{\text { finitely generated } S \text {-torsion free modules }\} .
\end{aligned}
$$

References: 3.1, 4.6, 4.7(c), 4.7(1), 5.1, and 5.2(a).

(7) Assume that $R$ is coherent from either side with finite left pure global dimension, and that $M$ is an $R$-bimodule which is finitely presented from either side. Then we may take

$$
\mathbb{X}=\operatorname{Add}\left(\operatorname{sub}\left(\operatorname{add}\left({ }_{R} M\right)\right)\right) .
$$

References: 3.1, 4.6, 4.7(d), 4.7(2), 5.1, and 5.2(a). 
Proposition 6.1. Assume that $R$ is right coherent. Then Flat $R$ is preenveloping, so it makes sense to consider the set of compact objects

$$
\mathcal{G}_{\text {Flat } R} \subseteq \mathrm{K}(\text { Flat } R)
$$

from Theorem 3.1. Then $\mathcal{G}_{\text {Flat } R}$ generates $\mathrm{K}($ Flat $R)$ if and only if Flat $R=\operatorname{Proj} R$.

Proof. That Flat $R$ is preenveloping over right coherent rings follows from [11, Prop. 6.5.1]. If Flat $R=\operatorname{Proj} R$, then we know from Section 6(1) above that

$$
\mathrm{K}(\text { Flat } R)=\mathrm{K}(\operatorname{Proj} R)
$$

is generated by $\mathcal{G}_{\text {Flat } R}=\mathcal{G}_{\operatorname{Proj} R}$. When Flat $R \neq \operatorname{Proj} R$ there exists a flat module $F$ which is not projective. Let

$$
\cdots \longrightarrow P_{2} \longrightarrow P_{1} \longrightarrow P_{0} \longrightarrow F \longrightarrow 0
$$

be an augmented projective resolution of $F$, and note that $(*)$ is pure exact but not split. Therefore Theorem 3.1 implies that $\mathcal{G}_{\text {Flat } R}$ does not generate $\mathrm{K}($ Flat $R$ ).

Of course, Proposition 6.1 above does not rule out the possibility that $\mathrm{K}(\mathrm{Flat} R$ ) could be generated by some larger set of compact objects than $\mathcal{G}_{\text {Flat } R}$. Hence we pose the following:

Question. When is $\mathrm{K}($ Flat $R$ ) compactly generated?

\section{Acknowledgements}

We sincerely thank Christian U. Jensen for his willingness to answer questions about pure global dimension, and in particular for pointing out Example 5.7(7).

\section{References}

[1] L. Angeleri-Hügel, Covers and envelopes via endoproperties of modules, Proc. London Math. Soc. 86 (2003), no. 3, 649-665.

[2] M. Auslander, Anneaux de Gorenstein, et torsion en algèbre commutative, Séminaire d'Algèbre Commutative dirigé par Pierre Samuel, 1966/67. Texte rédigé, d'après des exposés de Maurice Auslander, Marquerite Mangeney, Christian Peskine et Lucien Szpiro. École Normale Supérieure de Jeunes Filles, Secrétariat mathématique, Paris, 1967.

[3] M. Auslander and M. Bridger, Stable module theory, Mem. Amer. Math. Soc., no. 94, Amer. Math. Soc., Providence, R.I., 1969.

[4] M. Auslander, I. Reiten and S. O. Smalø, Representation theory of Artin algebras, Cambridge Stud. Adv. Math., vol. 36, Cambridge Univ. Press, Cambridge, 1995.

[5] L. L. Avramov and A. Martsinkovsky, Absolute, relative, and Tate cohomology of modules of finite Gorenstein dimension, Proc. London Math. Soc. 85 (2002), no. 2, 393-440. 
[6] D. Baer, H. Brune, and H. Lenzing, A homological approach to representations of algebras. II. Tame hereditary algebras, J. Pure Appl. Algebra 26 (1982), no. 2, 141-153.

[7] L. W. Christensen, Gorenstein dimensions, Lecture Notes in Math., vol. 1747, Springer-Verlag, Berlin, 2000.

[8] L. W. Christensen, A. Frankild and H. Holm, On Gorenstein projective, injective and flat dimensions - a functorial description with applications, J. Algebra 302 (2006), no. 1, 231-279.

[9] W. Crawley-Boevey, Locally finitely presented additive categories, Comm. Algebra 22 (1994), no. 5, 1641-1674.

[10] E. E. Enochs and O. M. G. Jenda, Balanced functors applied to modules, $J$. Algebra 92 (1985), no. 2, 303-310.

[11] E. E. Enochs and O. M. G. Jenda, Relative homological algebra, de Gruyter Exp. Math., vol. 30, Walter de Gruyter \& Co., Berlin, 2000.

[12] E. E. Enochs, O. M. G. Jenda and B. Torrecillas, Gorenstein flat modules, Nanjing Daxue Xuebao Shuxue Bannian Kan 10 (1993), no. 1, 1-9.

[13] E. E. Enochs, O. M. G. Jenda and J. Xu, Foxby duality and Gorenstein injective and projective modules, Trans. Amer. Math. Soc. 348 (1996), no. 8, 3223-3234.

[14] P. Gabriel, Des catégories abéliennes, Bull. Soc. Math. France 90 (1962), 323-448.

[15] W. Geigle, The Krull-Gabriel dimension of the representation theory of a tame hereditary Artin algebra and applications to the structure of exact sequences, Manuscripta Math. 54 (1985), no. 1-2, 83-106.

[16] K. R. Goodearl, Ring theory. Nonsingular rings and modules, Pure Appl. Math., vol. 33, Marcel Dekker Inc., New York, 1976.

[17] L. Gruson and C. U. Jensen, Modules algébriquement compacts et foncteurs $\lim ^{(i)}$, C. R. Acad. Sci. Paris Sér. A-B 276 (1973), A1651-A1653.

[18] H. Holm, Gorenstein homological dimensions, J. Pure Appl. Algebra 189 (2004), no. 1-3, 167-193.

[19] S. Iyengar and H. Krause, Acyclicity versus total acyclicity for complexes over noetherian rings, Doc. Math. 11 (2006), 207-240.

[20] C. U. Jensen and H. Lenzing, Algebraic compactness of reduced products and applications to pure global dimension, Comm. Algebra 11 (1983), no. 3, 305-325.

[21] C. U. Jensen and H. Lenzing, Model-theoretic algebra with particular emphasis on fields, rings, modules, Algebra Logic Appl., vol. 2, Gordon and Breach Science Publishers, New York, 1989.

[22] P. Jørgensen, Existence of Gorenstein projective resolutions and Tate cohomology, J. Eur. Math. Soc. 9 (2007), 59-76.

[23] P. Jørgensen, The homotopy category of complexes of projective modules, Adv. Math. 193 (2005), no. 1, 223-232. 
[24] R. Kiełpiński and D. Simson, On pure homological dimension, Bull. Acad. Polon. Sci. Sér. Sci. Tech. 23 (1975), 1-6.

[25] H. Krause, The stable derived category of a Noetherian scheme, Compos. Math. 141 (2005), no. 5, 1128-1162.

[26] T.-Y. Lam, Lectures on modules and rings, Grad. Texts in Math., vol. 189, Springer-Verlag, New York, 1999.

[27] H. Lenzing, Homological transfer from finitely presented to infinite modules, Abelian group theory (Honolulu, Hawaii, 1983), Lecture Notes in Math., vol. 1006, Springer, Berlin, 1983, pp. 734-761.

[28] S. Mac Lane, Categories for the working mathematician, second ed., Grad. Texts in Math., vol. 5, Springer-Verlag, New York, 1998.

[29] A. Neeman, Triangulated categories, Ann. of Math. Stud., vol. 148, Princeton Univ. Press, Princeton, NJ, 2001.

[30] B. L. Osofsky, Homological dimensions of modules, Conference Board of the Mathematical Sciences Regional Conference Series in Mathematics, no. 12., Amer. Math. Soc., Providence, R. I., 1973.

[31] J. J. Rotman, Notes on homological algebras, Van Nostrand Reinhold Math. Stud., vol. 26, Van Nostrand Reinhold Co., New York, 1970.

[32] R. Takahashi, On the category of modules of Gorenstein dimension zero, Math. Z. 251 (2005), no. 2, 249-256.

[33] C. A. Weibel, An introduction to homological algebra, Cambridge Stud. Adv. Math., vol. 38, Cambridge Univ. Press, Cambridge, 1994.

Henrik Holm holm@imf.au.dk

Department of Mathematical Sciences

University of Aarhus

Ny Munkegade, Building 1530, DK-8000 Aarhus C

Denmark

http://home.imf.au.dk/holm/

Peter Jørgensen peter.jorgensen@ncl.ac.uk

School of Mathematics and Statistics

Newcastle University

Newcastle upon Tyne NE1 7RU

United Kingdom

http://www.staff.ncl.ac.uk/peter.jorgensen/

This article is available at http://intlpress.com/HHA/v9/n1/a11 